\title{
BMJ Open Comparison of delivery catheter-based and stylet-based right ventricular lead placement at the right ventricular septum under fluoroscopic guidance judged by cardiac CT (Mt. FUJI): a study protocol for the Mt. FUJI randomised controlled trial
}

\author{
Yoshihisa Naruse (i) , ${ }^{1}$ Keisuke Miyajima, ${ }^{2}$ Ryo Sugiura, ${ }^{3}$ Masahiro Muto, ${ }^{4}$ \\ Michio Ogano, ${ }^{5}$ Nobutake Kurebayashi, ${ }^{6}$ Tomoyuki Shiozawa, ${ }^{7}$ Yumi Kiyama, ${ }^{8}$ \\ Eiko Nagata, ${ }^{8}$ Keiichi Odagiri, ${ }^{8}$ Yuichiro Maekawa, ${ }^{1}$ Mt. FUJI trial investigators
}

To cite: Naruse Y, Miyajima K, Sugiura $\mathrm{R}$, et al. Comparison of delivery catheter-based and stylet-based right ventricular lead placement at the right ventricular septum under fluoroscopic guidance judged by cardiac CT (Mt. FUJI): a study protocol for the Mt. FUJl randomised controlled trial. BMJ Open 2021;11:e046782. doi:10.1136/ bmjopen-2020-046782

- Prepublication history for this paper is available online. To view these files, please visit the journal online (http://dx.doi org/10.1136/bmjopen-2020046782).

Received 12 November 2020 Accepted 10 May 2021

Check for updates

(c) Author(s) (or their employer(s)) 2021. Re-use permitted under CC BY-NC. No commercial re-use. See rights and permissions. Published by BMJ.

For numbered affiliations see end of article.

Correspondence to Dr Yoshihisa Naruse; ynaruse@hama-med.ac.jp

\section{ABSTRACT}

Introduction Pacing-induced cardiomyopathy occasionally occurs in patients undergoing pacemaker implantation. Although compared with right ventricular (RV) apical pacing, RV septal pacing can attenuate left ventricular dyssynchrony; the success rate of lead placement on the RV septum using the stylet system is low. Additionally, no randomised controlled trial has addressed the issue regarding the accuracy of RV lead placement on the RV septum using the stylet and delivery catheter systems. This study hypothesises that a newly available delivery catheter system can improve the accuracy of RV lead placement on the RV septum. Methods and analysis In a multicentre, prospective, randomised, single-blind, controlled trial, 70 patients with pacemaker indication owing to atrioventricular block will be randomised to either the delivery catheter or stylet group before the pacemaker implantation procedure. The position of the RV lead tip will be assessed using ECG-gated cardiac CT in all patients within 4 weeks after pacemaker implantation. Lead tip positions are classified into three groups: (1) RV septum, (2) anterior/ posterior edge of the RV septal wall and (3) RV free wall. The primary endpoint will be the success rate of RV lead tip placement on the RV septum, which will be evaluated using cardiac CT.

Ethics and dissemination This study will be conducted according to the stipulations of the Helsinki Declaration and the institutional review board of Hamamatsu University School of Medicine. The results of the study will be disseminated at several research conferences and will be published in peer-reviewed journals.

Trial registration number jRCTs042200014; Pre-results.

\section{INTRODUCTION}

Pacemaker implantation is an established treatment option for patients with
Strengths and limitations of this study

This is the first multicentre randomised controlled trial to assess the superiority of the delivery catheter system over the stylet system for placing the tip of the right ventricle (RV) lead on the RV septum.

- All lead tip positions will be confirmed using ECGgated CT.

- The inability of the study to show the prognostic benefit of the delivery catheter system over the stylet system is a limitation.

bradycardia. Pacing-induced cardiomyopathy occasionally occurs in patients undergoing pacemaker implantation owing to atrioventricular block (AVB) and was associated with reduced left ventricular (LV) ejection fraction, increased risk of heart failure hospitalisation, new-onset atrial fibrillation or all-cause mortality. ${ }^{1}$ Although theoretically, right ventricular (RV) septal pacing can attenuate LV dyssynchrony more than RV apical pacing, ${ }^{2}$ the non-apical RV pacing site did not show any benefit in terms of the incidence of pacing-induced cardiomyopathy over the apical lead position. ${ }^{3-6}$ A serious problem with the strategy of RV septal pacing is the low success rate of placement of the RV lead on the RV septum, confirmed using CT. ${ }^{7-11}$ Fluoroscopic left anterior oblique (LAO) view was used as an indicator of successful RV lead placement on the RV septum. However, thoracic CT revealed that only 16\%-76\% (median $41 \%$ (IQR 21\%-43\%)) of the RV 
leads were successfully anchored on the RV septum despite successful fluoroscopic placement of the RV leads on the RV septum. ${ }^{7-11}$ A recent report suggested that unexpected free wall pacing leads to increased occurrence of cardiovascular death and heart failure hospitalisation in patients undergoing RV septal pacing owing to AVB. ${ }^{7}$ Recent reports showed that the use of individualised $\mathrm{LAO}^{12}$ or right ventriculography ${ }^{13}$ could improve the CT-judged accuracy of RV lead deployment on the RV septum by over $90 \%$. However, determining the individualised LAO or performing right ventriculography during the procedure could prolong the procedural and fluoroscopic time. Therefore, simple methods to improve the success rate of RV lead implantation on the RV septum are needed. Although for decades, the stylet system was only available for lead placement, a new delivery catheter system was recently designed for pacemaker implantation. Recently, a single-centre retrospective study revealed that delivery catheter system achieved the RV lead placement on RV septum more frequently than stylet system. ${ }^{14}$ However, no randomised controlled trial has examined the accuracy of RV lead placement on the RV septum using the stylet system versus using the delivery catheter system. Therefore, the comparison of delivery catheterbased and stylet-based RV lead placement at the RV septum under fluoroscopic guidance judged by cardiac CT (Mt FUJI study) was compared with investigate whether, compared with using the stylet system, using the delivery catheter system would lead to increased success rate of RV lead placement on the RV septum in patients undergoing pacemaker implantation owing to AVB.

\section{METHODS AND ANALYSIS \\ Study design}

The Mt FUJI study is a multicentre, prospective, randomised, single-blind, controlled trial for comparing the accuracy of RV lead anchoring on the RV septum between the delivery catheter and stylet systems. Patients will be recruited from seven tertiary centres (Hamamatsu University School of Medicine (HUSM), Seirei Mikatahara General Hospital, Seirei Hamamatsu General Hospital, Hamamatsu Medical Center, Shizuoka Medical Center, Chutoen General Medical Center and Juntendo University Shizuoka Hospital). HUSM is the sponsor of the study, with financial support from Medtronic Japan Co, Ltd and HUSM Grant-in-Aid. To date (October 2020), 49 patients have been enrolled, and the trial intends to recruit 70 patients. The first implantation occurred in June 2020, and the study (including patient enrolment, follow-up and data analysis) will last 12 months. The Mt FUJI trial is registered on the website (https://jrct.niph. go.jp/en-latest-detail/jRCTs042200014).

\section{Objectives}

The primary objective of the Mt FUJI trial is to assess the success rate of RV lead-tip placement on the RV septum evaluated using cardiac CT.

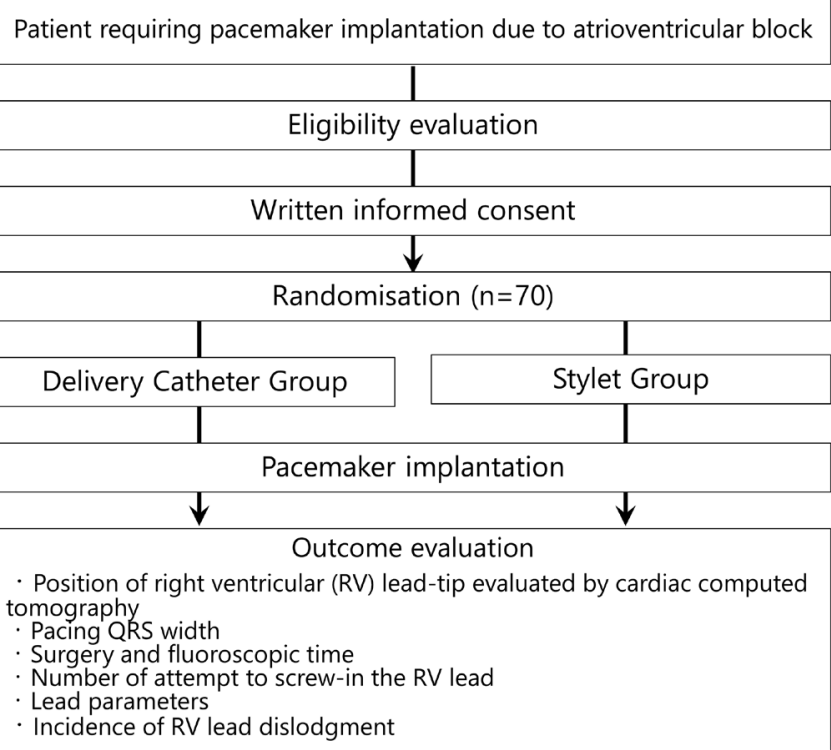

Figure 1 Study design and flow. AV, atrioventricular.

The secondary objectives of the Mt FUJI trial are as follows: to determine (1) paced QRS width; (2) surgery time; (3) fluoroscopic time; (4) number of attempts to screw in the RV lead; (5) RV lead parameters, including R-wave amplitude, pacing threshold and impedance; and (6) the incidence of RV lead dislodgment at discharge.

\section{Patient population, inclusion and exclusion criteria}

Investigators will screen consecutive patients using the inclusion/exclusion criteria, which are based on age, pacemaker indication and cardiovascular medical history. Patients with a class I or II indication for pacemaker owing to AVB, ${ }^{15}$ who are older than 20 years, and provided written informed consent will be invited to participate in the study. The exclusion criteria are as follows: those with (1) severely impaired LV function, defined as LV ejection fraction $<35 \%$; (2) end-stage renal failure on haemodialysis; (3) prior open heart surgery; (4) persistent atrial fibrillation; and (5) congenital heart disease (figure 1).

\section{Randomisation and intervention}

Eligible patients who agree to participate in the study will be randomised in a 1:1 ratio to either the delivery catheter group or the stylet group before the pacemaker implantation procedure. Randomisation will be balanced with respect to age, gender and participating hospitals and will be performed using a web-based randomisation system (UMIN INDICE cloud: https://www.umin.ac.jp/ indice/cloud.html), based on a minimisation scheme. In patients allocated to the delivery catheter group, a delivery catheter (C315 series, Medtronic, Minneapolis, Minessota, USA) and delivery catheter-compatible leads (SelectSecure Model 3830, Medtronic) will be used. C315-HIS is recommended for use at the beginning, and other delivery catheters such as C315-S5 and C315S10 can be used as a second choice. In the stylet group, conventional stylet-based RV leads will be used (TENDRIL 
A

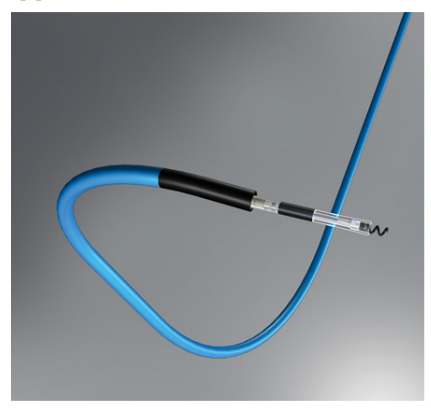

B

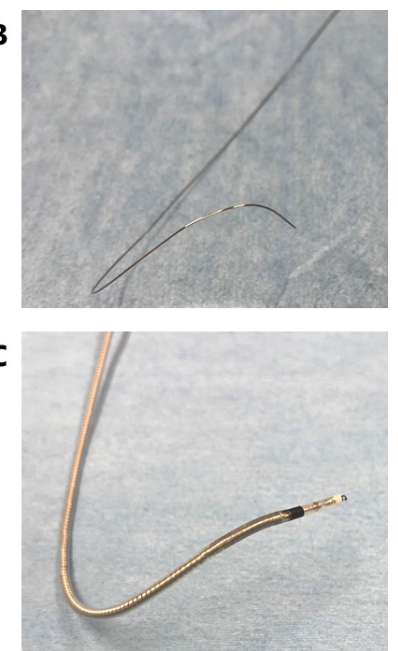

lead is intended to be anchored on the second or third quadrant. ${ }^{10}$

In the stylet group, the choice of a stylet, that is, a conventional manually shaped stylet, a steerable stylet (Locator, Abbott) or a preshaped stylet (Ez stylet, Japan Lifeline, Tokyo, Japan), is left to the discretion of the attending physician. In the delivery catheter group, the delivery catheter is guided to the RV over the 0.035-inch guidewire and is directed to the RV septum. After the delivery catheter is positioned appropriately, the RV lead is passed through the delivery catheter to the RV septum. The position of the tip of the RV lead is confirmed using $\mathrm{X}$-ray fluoroscopy in the LAO and RAO views. Once the RV lead tip is attached to the RV septum, the capture threshold and $\mathrm{R}$ wave amplitudes are measured using a unipolar tip. When the screw is deployed, the capture thresholds, R-wave amplitudes and lead impedances are assessed. After confirming that these values are good, the delivery catheter is peeled away. Crossover between delivery catheters and stylets is allowed based on the operator's decision if the placement of the RV lead using the allocated device is difficult.

\section{Outcome measures}

For the primary outcome measure, electrocardiography (ECG)-gated cardiac CT will be performed in all patients within 4 weeks after pacemaker implantation. The use of contrast medium is recommended unless study patients have renal dysfunction or allergic diseases, such as bronchial asthma. CT scans are analysed in the axial view or multiplanar-reconstructed coronal, sagittal, short-axis, long-axis and four-chamber views. Two trained investigators with no knowledge of clinical outcomes will independently evaluate CT scans. When there is interobserver disagreement, a third doctor from a different facility is consulted. Lead tip positions are classified into three groups: (1) RV septum, (2) anterior/posterior edge of the RV septal wall and (3) RV free wall (figure 3). The primary endpoint will be the success rate of RV lead tip placement on the RV septum evaluated using cardiac CT. Intention-to-treat analyses will be performed between the delivery catheter and stylet groups. If RV lead dislodgment occurs before ECG-gated cardiac CT is performed, the patients will be considered to have unsuccessful RV lead placement on the RV septum, irrespective of the result of a reimplantation procedure.

Pacing QRS width is assessed by a physician who is not involved in the pacemaker implantation procedure. Surgery time is defined as the time from skin incision to skin closure, and fluoroscopic time will be obtained from the fluoroscopy system. The number of attempts to screw-in the RV lead will be counted during the pacemaker implantation procedure. RV lead parameters, including R-wave amplitude, pacing threshold and impedance, will be measured using a pacemaker programmer. RV lead dislodgment at discharge is defined, as follows: (1) obvious lead dislocation or lead perforation assessed using chest roentgenography that requires reoperation, ularly to its contour into quadrants. The tip of the RV 
A

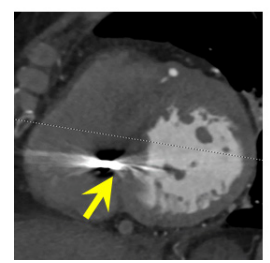

B

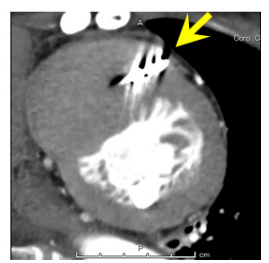

C

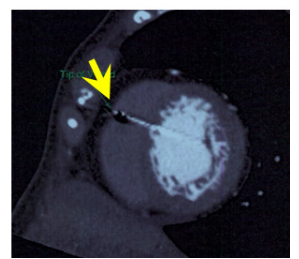

Figure 3 The position of the tip of the right ventricular (RV) lead assessed by electrocardiography-gated cardiac CT. Multiplanar-reconstructed short-axis views show that the tip of the RV leads are positioned at the (A) RV septum, (B) anterior edge of the RV septal wall and (C) RV free wall.

(2) pacing threshold of $>3.0 \mathrm{~V}$ with a nominal pulse width or (3) $\mathrm{R}$ wave amplitude of $<1.0 \mathrm{mV}$.

\section{Data collection and monitoring}

A physician or a clinical research coordinator will collect information on demographic characteristics during enrolment, periprocedural variables and variables after pacemaker implantation until discharge. All data will be stored anonymously and will be locked once collection is complete. The investigators must follow the instructions of this study protocol. They cannot modify the protocol without permission from the ethics committee and must record any deviations from the protocol.

The data monitoring committee, which is independent of the investigators, will perform the central monitoring of the data. All adverse events related to the interventions will be recorded and monitored until resolved. Medical personnel will decide whether continued trial participation is feasible based on these reports, with the participant making the final decision.

\section{Sample size estimation}

According to a previous observational study, the success rate of $\mathrm{RV}$ lead placement on the RV septum ranges from $16 \%-76 \%$ (median $41 \%$ (IQR 21\%-43\%)) in the stylet system. ${ }^{7-11}$ Although our single-centre retrospective study has recently described that the success rate of RV septal lead placement using a delivery catheter group was $100 \%$, no randomised controlled study is available about the accuracy of anchoring RV lead on RV septum. The success rate of left bundle branch pacing using a delivery catheter has been reported to be $88 \% .{ }^{16}$ The success rate of RV septal lead placement using a delivery catheter is expected to be as high as that of left bundle branch pacing. Therefore, the prevalence of successful anchoring of the RV lead tip on the RV septum is estimated to be $85 \%$ in the delivery catheter group and $50 \%$ in the stylet group. A sample size of 70 patients (35 patients in the delivery catheter group and 35 patients in the stylet group) will be selected to obtain an $80 \%$ power and a CI of $95 \%$ for detecting differences between the two groups, after making allowance for $5 \%$ dropout after randomisation.

\section{Statistical analysis}

Continuous variables will be expressed as mean \pm SD or median (IQR) and will be compared using the unpaired t-test or Mann-Whitney U-test, according to data distribution. All categorical variables will be expressed as raw numbers and percentages and will be analysed using the Fisher's exact test. A two-tailed $\mathrm{p}$ value of $<0.05$ will be considered statistically significant. All analyses will be performed using R program V.3.6.3 (The R Foundation for Statistical Computing, Vienna, Austria).

\section{Patient and public involvement}

No formal patient or public organisations were set up, and there was no patient or public involvement in the decision regarding the study design and in the planning of the study.

\section{ETHICS AND DISSEMINATION}

\section{Ethical approval and informed consent}

All study activities are coordinated by the clinical research centre at HUSM in Hamamatsu, Shizuoka, Japan. This study protocol was approved by the clinical research review board of the HUSM (approval number: C0102019) and by the hospital administrator in all hospitals. The study was conducted according to the guidelines of the Declaration of Helsinki and Clinical Trials Act. Written informed consent was obtained from all patients or their legal guardians before inclusion and randomisation. The research coordinator will describe the study to the prospective participant and answers any questions. Patients are reminded of the confidentiality of the study process and of their freedom to withdraw at any time, without explanations or effects on their future interactions with their healthcare provider or employer. If the individual is willing to participate, he or she is asked to sign an informed consent document, which the research coordinator cosigns.

\section{Data statement}

Data from Mt FUJI study are available on reasonable request.

\section{Dissemination plan}

Trial results will be communicated at a local level to healthcare professionals and patient involvement groups. The results of this study will be disseminated at several research conferences and will be published in international peer-reviewed journals, with authorship determined by the International Committee of Medical Journal Editors' guidelines. Medtronic Inc will be informed prior to publication, but no publication restrictions apply.

\section{DISCUSSION}

The Mt FUJI study is a multicentre, prospective, randomised, single-blind, controlled trial for investigating whether the use of a delivery catheter system, compared with the use of a stylet system, would lead to 
an increased success rate of RV lead placement on the RV septum in patients undergoing pacemaker implantation owing to AVB. Although pacemaker is commonly the first choice treatment in patients with bradycardia, $\mathrm{RV}$ pacing-induced cardiomyopathy remains one of the chronic adverse events in patients undergoing pacemaker implantation. Robust and reproducible methods for achieving accurate anchoring of the RV lead tip on the RV septum are needed before discussions on the clinical efficacy of RV septal pacing over RV apical pacing for reducing RV pacing-induced cardiomyopathy. The weakness of the stylet system may be attributed to the weakness of the back-up force and the flexible design of the lead body to avoid perforation of leads. Since the surface of the RV septum is smooth, the tip of RV lead could slip towards the anterior edge of RV septum when pushing the RV lead with stylet before screw-in. Delivery catheter system that has stronger back-up force than stylet system and always points to the interventricular septum due to their fixed three-dimensional shape could overcome this technical difficulty. Once the Mt FUJI trial proves that our hypothesis is true, the delivery catheter system would become the first choice for achieving RV septal pacing, and further studies would be planned to assess the beneficial effect of RV septal pacing using a delivery catheter system to reduce RV pacing-induced cardiomyopathy compared with RV apical pacing.

\section{Author affiliations}

${ }^{1}$ Division of Cardiology, Internal Medicine III, Hamamatsu University School of Medicine, Hamamatsu, Japan

${ }^{2}$ Department of Cardiology, Seirei Mikatahara Hospital, Hamamatsu, Japan

${ }^{3}$ Department of Cardiology, Seirei Hamamatsu Hospital, Hamamatsu, Japan

${ }^{4}$ Department of Cardiology, Hamamatsu Medical Center, Hamamatsu, Japan ${ }^{5}$ Department of Cardiovascular Medicine, Shizuoka Medical Center, Sunto-gun, Japan

${ }^{6}$ Department of Cardiology, Chutoen General Medical Center, Kakegawa, Japan

${ }^{7}$ Department of Cardiology, Juntendo Shizuoka Hospital, Izunokuni, Japan

${ }^{8}$ Centre for Clinical Research, Hamamatsu University School of Medicine Hospital, Hamamatsu, Japan

Acknowledgements We would like to thank Editage (www.editage.com) for English language editing.

Collaborators Mt. FUJI trial investigators: Yutaro Kaneko, Tomoaki Sakakibara, Taro Narumi, Makoto Sano, Satoshi Mogi, Kenichiro Suwa, Hayato Ohtani, Masao Saotome, Tuyoshi Urushida, Akira Mizukami, Hideyuki Hasebe, Keisuke Iguchi, Akiko Atsumi, Naoya Inoue, Tomoya Iwawaki, Tomotaka Suzuki, Takashi Ogane, Naoki Tsurumi, Yumiko Joko, Shuji Morikawa, Satoru Suwa, Manabu Ogita, Hideki Wada, Shintaro Takano, Jun Shitara, Shoichiro Yatsu, Taketo Sonoda, Kentaro Yasuda, Ryota Nishio, Daigo Takahashi, Go Ishikawa, Soushi Moriya, Kei Kimura, Jun Tanabe, Kohei Sawasaki, Yohei Takayama, Natsuko Hosoya, Yasushi Wakabayashi, Yoshitaka Kawaguchi, Tomoyuki Watanabe, Yasuyo Takashima, Ayako Okazaki, Kazuki Ito, Ryuta Henmi, Daichi Isomura, Hideki Saito and Yoshinobu Kato.

Contributors YN, YK, EN and KO contributed to the conception and design of this work. KM, RS, MM, MO, NK and TS contributed to data collection. YN contributed to the analysis and interpretation of the data and drafting of the manuscript. MO, TS and $\mathrm{KO}$ contributed to critical revision of the manuscript for important intellectual content. YM contributed to the final approval of the manuscript.

Funding Hamamatsu University School of Medicine (HUSM) sponsored the study, with financial support from Medtronic Japan Co Ltd (grant number: ERP-201912060) and HUSM Grant-in-Aid (grant number: N/A).

Competing interests YM received research and fellowship grants from Biotronik, Medtronic and Abbott. There is no conflict of interest for the other authors.
Patient and public involvement Patients and/or the public were not involved in the design, or conduct, or reporting, or dissemination plans of this research.

Patient consent for publication Not required.

Provenance and peer review Not commissioned; externally peer reviewed.

Open access This is an open access article distributed in accordance with the Creative Commons Attribution Non Commercial (CC BY-NC 4.0) license, which permits others to distribute, remix, adapt, build upon this work non-commercially, and license their derivative works on different terms, provided the original work is properly cited, appropriate credit is given, any changes made indicated, and the use is non-commercial. See: http://creativecommons.org/licenses/by-nc/4.0/.

ORCID iD

Yoshihisa Naruse http://orcid.org/0000-0001-9630-951X

\section{REFERENCES}

1 Dor O, Haim M, Barrett O, et al. Incidence and clinical outcomes of pacing induced cardiomyopathy in patients with normal left ventricular systolic function and atrioventricular block. Am J Cardiol 2020;128:174-80.

2 Inoue K, Okayama H, Nishimura K, et al. Right ventricular septal pacing preserves global left ventricular longitudinal function in comparison with apical pacing: analysis of speckle tracking echocardiography. Circ J 2011;75:1609-15.

3 Bansal R, Parakh N, Gupta A, et al. Incidence and predictors of pacemaker-induced cardiomyopathy with comparison between apical and non-apical right ventricular pacing sites. J Interv Card Electrophysiol 2019;56:63-70.

4 Mizukami A, Matsue Y, Naruse Y, et al. Implications of right ventricular septal pacing for medium-term prognosis: Propensitymatched analysis. Int J Cardiol 2016;220:214-8.

5 Kaye GC, Linker NJ, Marwick TH, et al. Effect of right ventricular pacing lead site on left ventricular function in patients with highgrade atrioventricular block: results of the Protect-Pace study. Eur Heart J 2015;36:856-62.

6 Spath NB, Wang K, Venkatasumbramanian S, et al. Complications and prognosis of patients undergoing apical or septal right ventricular pacing. Open Heart 2019;6:e000962.

7 Hattori M, Naruse Y, Oginosawa Y, et al. Prognostic impact of lead tip position confirmed via computed tomography in patients with right ventricular septal pacing. Heart Rhythm 2019;16:921-7.

8 Sharma G, Salahuddin S, Sanders P, et al. Inadequacy of fluoroscopy and electrocardiogram in predicting septal position in RVOT pacing - Validation with cardiac computed tomography. Indian Heart J 2016;68:174-80.

9 Pang BJ, Joshi SB, Lui EH, et al. Validation of conventional fluoroscopic and ECG criteria for right ventricular pacemaker lead position using cardiac computed tomography. Pacing Clin Electrophysiol 2014;37:495-504.

10 Osmancik P, Stros P, Herman D, et al. The insufficiency of left anterior oblique and the usefulness of right anterior oblique projection for correct localization of a computed tomography-verified right ventricular lead into the midseptum. Circ Arrhythm Electrophysiol 2013;6:719-25.

11 Chen Y-L, Wang H-T, Chen $\mathrm{H}-\mathrm{C}$, et al. Localization of right ventricular non-apical lead position: comparison of three-dimensional echocardiography, computed tomography, and fluoroscopic imaging. $J$ Int Med Res 2021;49:030006052199615.

12 Squara F, Scarlatti D, Riccini P, et al. Individualized left anterior oblique projection: a highly reliable patient-tailored fluoroscopy criterion for right ventricular lead positioning. Circ Arrhythm Electrophysiol 2018;11:e006107.

13 Shimeno K, Yoshiyama T, Abe Y, et al. The usefulness of right ventriculography to aid anchoring a pacing lead to the right ventricular septum. Europace 2018;20:1154-60.

14 Miyajima K, Urushida T, Naruse Y. The usefulness of a delivery catheter system for right ventricular "true" septal pacing. Heart Vessels 2021 doi:10.1007/s00380-021-01780-8

15 Kusumoto FM, Schoenfeld MH, Barrett C. ACC/AHA/HRS guideline on the evaluation and management of patients with bradycardia and cardiac conduction delay: a report of the American College of Cardiology/American heart association Task force on clinical practice guidelines and the heart rhythm Society. Circulation 2018;2019:e382-482.

$16 \mathrm{Hu}$ Y, Li H, Gu M, et al. Comparison between his-bundle pacing and left bundle branch pacing in patients with atrioventricular block. $J$ Interv Card Electrophysiol 2020 doi:10.1007/s10840-020-00869-w 